\title{
ESTUDO DA REOLOGIA E DA SALINIDADE DE MICROEMULSÕES A BASE DE GLICERINA PARA SEREM UTILIZADAS NA RECUPERAÇÃO DE PETRÓLEO
}

\author{
A. B. CARVALHO ${ }^{*}$, A. G. CABRAL ${ }^{1}$, E. A. ARAUJO ${ }^{1}$, A. I. C. GARNICA ${ }^{1}$, F. D. S. \\ CURBELO $^{1 *}$ \\ ${ }^{1}$ Universidade Federal da Paraíba, Centro de Tecnologia, Departamento de Engenharia \\ Química \\ *E-mail para contato: amanda93carv@gmail.com, fabioladias@yahoo.com
}

\begin{abstract}
RESUMO - Os métodos convencionais de recuperação de petróleo apresentam desvantagens devido às baixas eficiências de deslocamento causadas pelas altas tensões interfaciais existentes entre os fluidos do reservatório. Os sistemas microemulsionados conseguem minimizar diferenças interfaciais entre estes fluidos, aumentando, com isso, o percentual de óleo recuperado. $\mathrm{O}$ presente trabalho teve como objetivo obter sistemas microemulsionados a partir de diagramas ternários com diferentes concentrações de $\mathrm{NaCl}$ (1, 2 e $3 \%$ em peso); analisar da influência do sal na região de microemulsão obtida e estudar as características reológicas desses sistemas. Para o desenvolvimento das microemulsões, foram utilizados um tensoativo não iônico (ultranex NP100), uma fase oleosa (óleo vegetal) e uma fase aquosa (solução de glicerina). A partir dos diagramas ternários obtidos, foi escolhido um ponto ternário na região de microemulsão em que se observou a presença de regiões de Winsor tipo II e tipo IV. Os ensaios reológicos foram realizados com faixa de rotação de 0 a $90 \mathrm{rpm}$, nas temperaturas de 30 a $70^{\circ} \mathrm{C}$, variando a cada $10^{\circ} \mathrm{C}$. Foi verificado que os sistemas microemulsionados estudados não sofreram influência do aumento da salinidade e, através dos ensaios reológicos, verificou-se, também, que estes sistemas, nas faixas de temperatura de 40 a $70^{\circ} \mathrm{C}$, foram classificados como fluidos newtonianos e apresentaram viscosidades adequadas a aplicações futuras em recuperação avançada de petróleo.
\end{abstract}

\section{INTRODUÇÃO}

Os métodos convencionais de recuperação de petróleo apresentam algumas desvantagens devido às baixas eficiências de deslocamento e, portanto, baixas recuperações, visto que o fluido injetado no reservatório não consegue deslocar o óleo do meio poroso devido às altas tensões interfaciais. (Lima, 2013).

Dentre os métodos especiais de recuperação, o método químico vem se mostrando muito eficiente, seja com a injeção de tensoativos ou de sistemas microemulsionados (Kumar e Mandal, 2016; Budhathoki et al., 2016). A utilização de sistemas microemulsionados consegue minimizar, de maneira significativa, as diferenças interfaciais entre água e óleo, o que melhora a eficiência de deslocamento do óleo no meio poroso (Thomas, 2004). 
Estimativas a respeito da porcentagem de óleo recuperado através de métodos convencionais têm conduzido a um fator de recuperação de cerca de $30 \%$, ou seja, de todo o óleo descoberto, apenas $30 \%$ pode ser recuperado por estes métodos (Thomas, 2004, Curbelo, 2007 e 2008), de modo que um grande percentual de óleo permanece retido no meio poroso.

Com isso, o objetivo dos métodos especiais é atuar na parcela correspondente a $70 \%$ do óleo, que é o volume percentual médio restante nos reservatórios após a recuperação convencional. Dessa forma, o estudo de sistemas microemulsionados realizados neste trabalho se faz importante na tentativa de melhorar a eficiência da recuperação de petróleo.

\section{METODOLOGIA}

\subsection{Materiais}

Para a construção dos diagramas ternários, foram utilizados: um tensoativo não iônico ultranex NP100 (U100) gentilmente cedido pela Oxiteno e utilizado sem purificação, um óleo vegetal como fase oleosa e a glicerina com 1,2 e $3 \%$ de $\mathrm{NaCl}$ em massa como fase aquosa.

\subsection{Obtenção dos diagramas ternários}

Para a construção do diagrama, foi utilizado $1 \mathrm{~g}$ de dois dos componentes, com proporções mássicas conhecidas e adicionados em um tubo de ensaio, em seguida, iniciou-se a titulação com o terceiro componente, até o surgimento de qualquer região, em que a massa do terceiro componente era determinada e, posteriormente, a fração mássica.

\subsection{Ensaio Reológico}

Os parâmetros reológicos foram determinados através do reômetro Brookfield LVDVIII Ultra. A amostra foi colocada no recipiente do reômetro e submetida a um torque suficiente para manter a rotação do spindle CP51 imerso na amostra. A faixa de rotação foi de 0 a 90 rpm, nas temperaturas de 30 a $70^{\circ} \mathrm{C}$, variando a cada $10^{\circ} \mathrm{C}$.

\section{RESULTADOS E DISCUSSÃO}

\subsection{Microemulsões}

As Figuras 1, 2 e 3 mostram os diagramas de fases obtidos para as concentrações de $\mathrm{NaCl}$ de 1, 2 e 3\%, respectivamente. Estas figuras apresentaram duas regiões: uma região de única fase microemulsionada (WIV) e outra região de duas fases, microemulsão e fase aquosa em excesso (WII). O aumento da concentração do sal (1, 2 e $3 \%$ de $\mathrm{NaCl}$ em massa), entretanto, não provocou mudanças significativas na região de microemulsão. Isso aconteceu, provavelmente, pelo fato do Ultranex NP100 ser um tensoativo não-iônico e apresentar baixa interação com os íons $\mathrm{Na}^{+}$e $\mathrm{Cl}^{-}$. 
Figura 1 - Diagrama ternário para o sistema 1: U100, óleo vegetal e glicerina 1\% $\mathrm{NaCl}$.

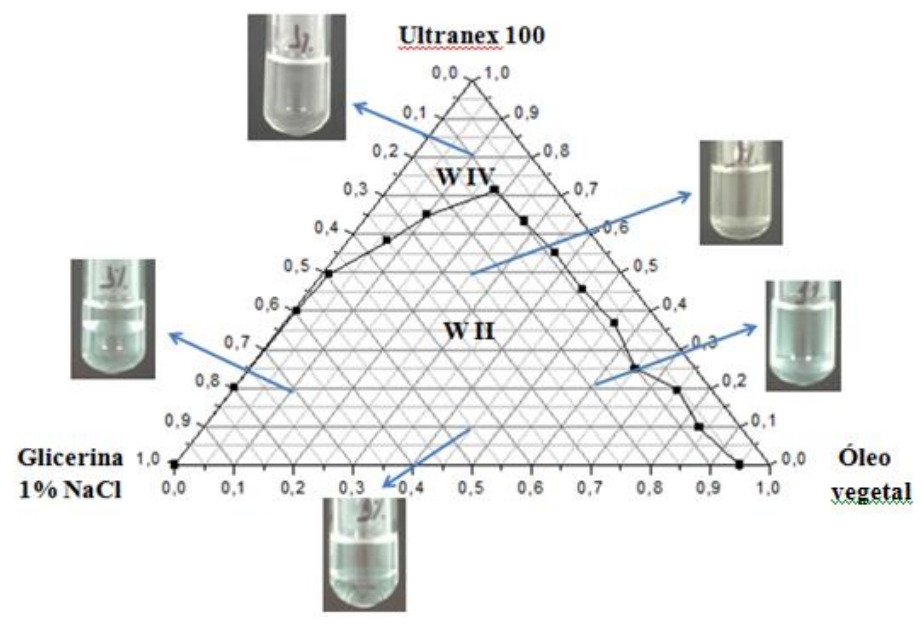

Figura 2 - Diagrama ternário para o sistema 2: U100, óleo vegetal e glicerina 2\% $\mathrm{NaCl}$.

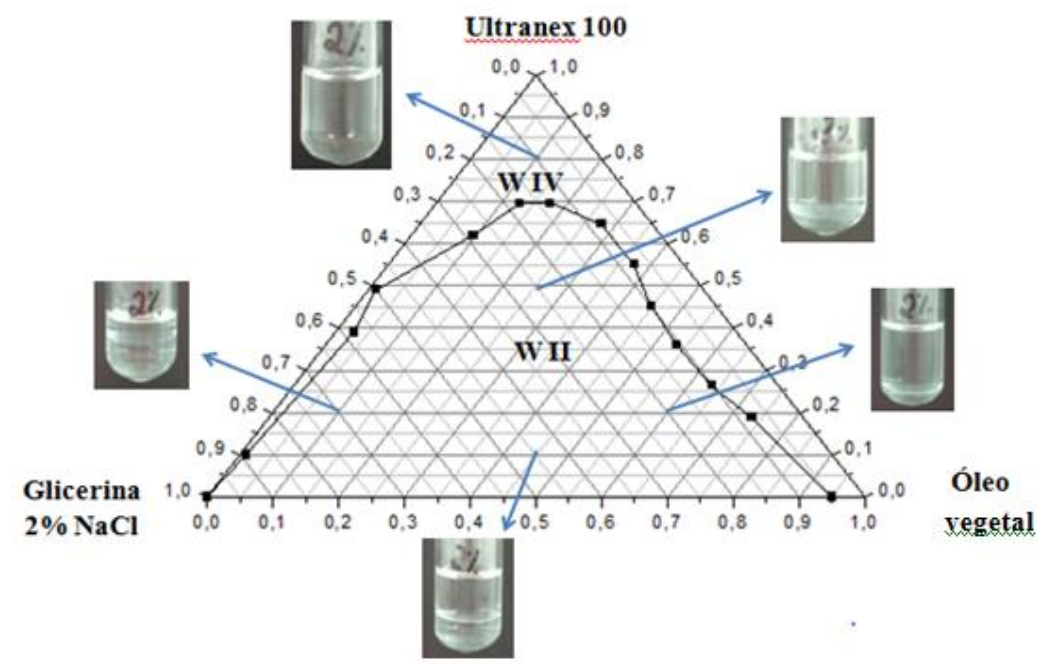

Figura 3 - Diagrama ternário para o sistema 3: U100, óleo vegetal e glicerina 3\% $\mathrm{NaCl}$.

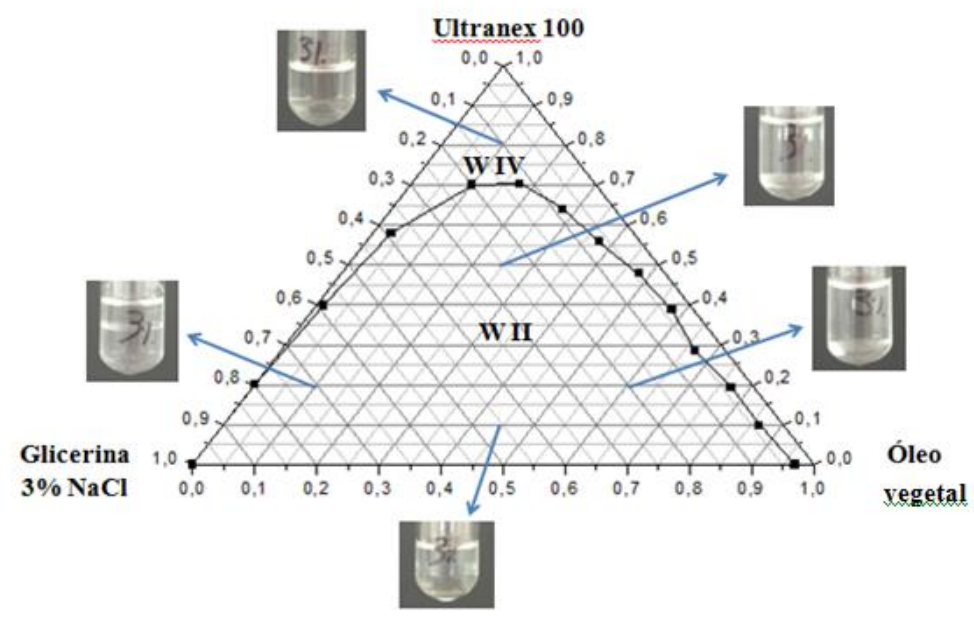




\subsection{Reologia}

O ponto ternário selecionado para o estudo reológico continha $80 \%$ de U100, $10 \%$ de óleo vegetal e $10 \%$ de glicerina com 1, 2 ou $3 \%$ em massa de $\mathrm{NaCl}$. As microemulsões com 1 , 2 e $3 \%$ de $\mathrm{NaCl}$ em fase aquosa são chamadas de sistemas 1, 2 e 3, respectivamente. Os resultados de viscosidade $\left(10^{-3} \mathrm{~Pa} . \mathrm{s}\right)$ em função da taxa de cisalhamento (1/s) para estes sistemas estão representados nas Figura 4 a 6.

Figura 4 - Log da viscosidade versus Log da taxa de Cisalhamento para sistema 1.

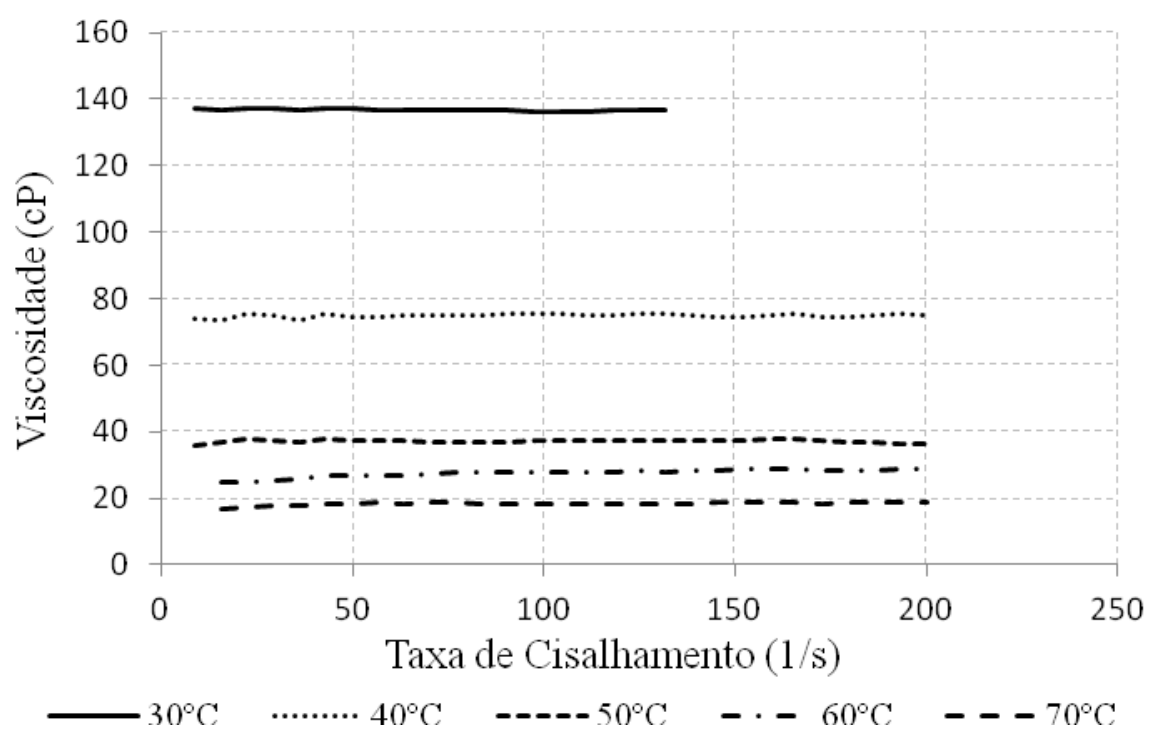

Figura 5 - Log da viscosidade versus Log da taxa de Cisalhamento para sistema 2.

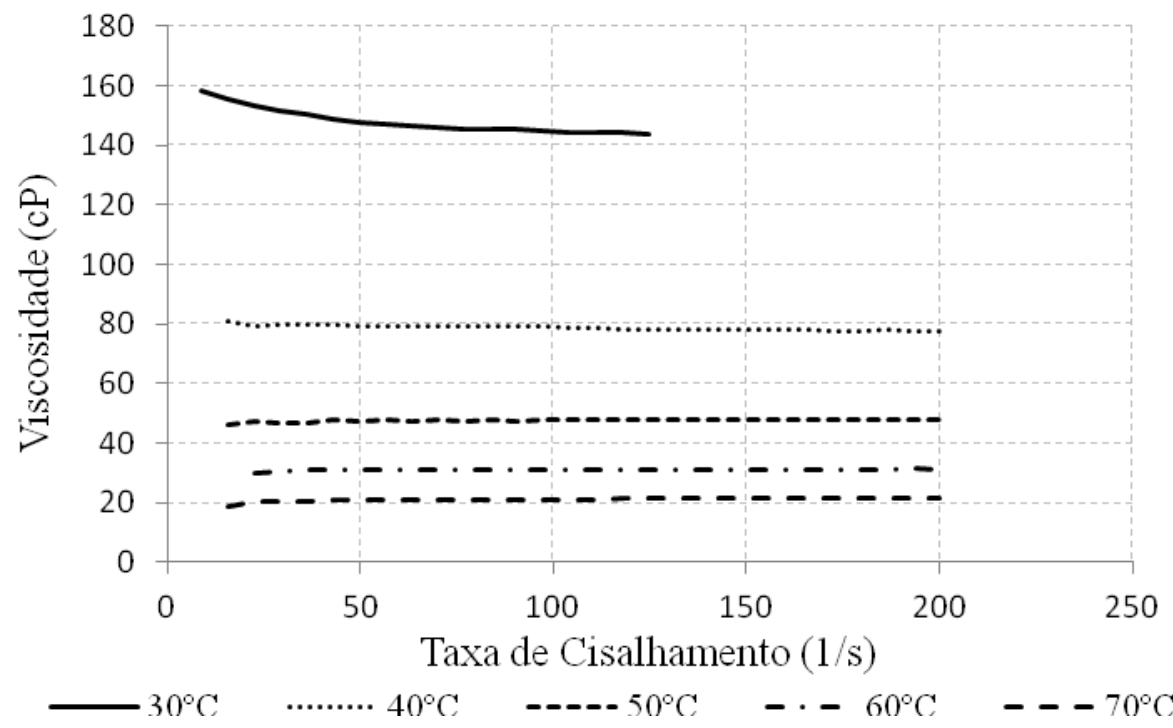


Figura 6 - Log da viscosidade versus Log da taxa de Cisalhamento para sistema 3.

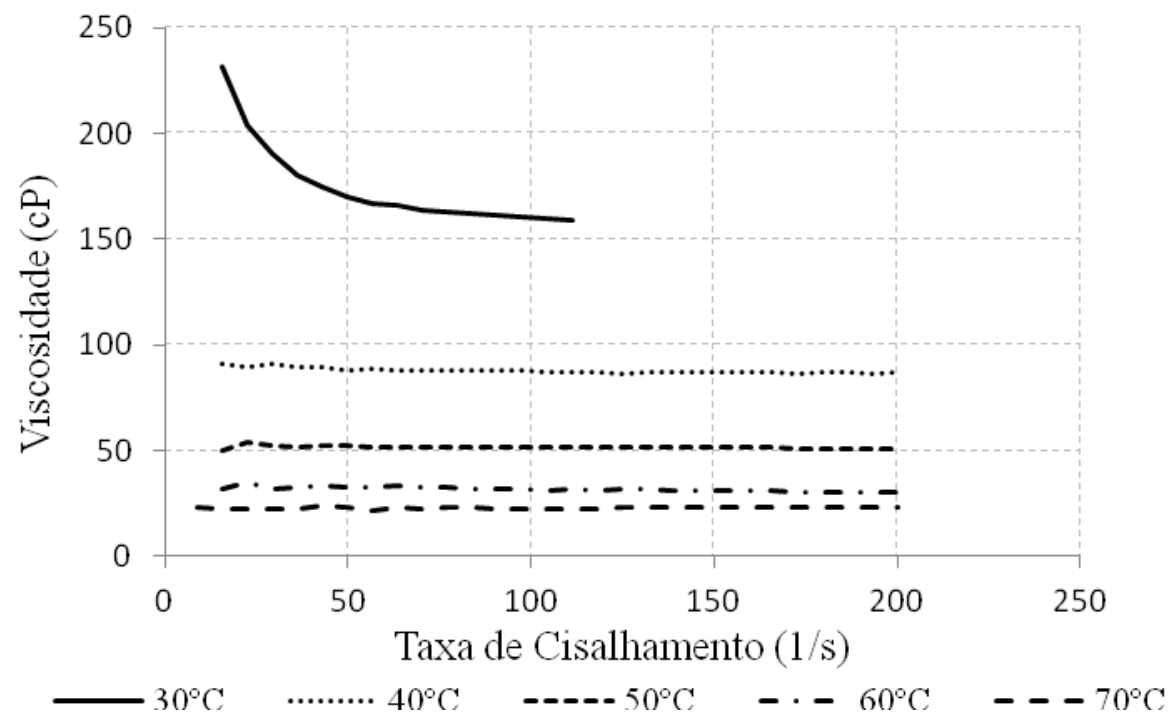

Conforme os dados apresentados nas Figuras 4, foi possível classificar o fluido do sistema 1 como newtonianos em todas as temperaturas avaliadas $\left(30,40,50,60\right.$ e $\left.70^{\circ} \mathrm{C}\right)$. Já para os sistema 2 e 3, conforme as Fig. 5 e 6, o fluido foi classificado como não-newtoniano (pseudoplástico) para a temperatura de $30^{\circ} \mathrm{C}$; ajustando-se ao modelo de Herschel Bulkley, e newtoniano para as temperaturas de $40,50,60$ e $70^{\circ} \mathrm{C}$.

Os termos da equação de Herschel-Buclkley para os sistemas 2 e 3 a $30^{\circ} \mathrm{C}$ estão representados na Tabela 5 .

Tabela 5. Termos da equação de Herschel-Bulkley para os sistemas 2 e 3 a $30^{\circ} \mathrm{C}$.

\begin{tabular}{|c|c|c|c|c|}
\hline & $\mathbf{R}^{\mathbf{2}}$ & $\tau_{0}\left(\mathbf{N} / \mathbf{m}^{\mathbf{2}}\right)$ & $\mathbf{n}$ & $\mathbf{K}$ \\
\hline Sistema 2 & 1,0 & $1,0 \times 10^{-2}$ & 0,9545 & 1,76 \\
\hline Sistema 3 & 0,9979 & $8,0 \times 10^{-1}$ & 0,92 & 2,15 \\
\hline
\end{tabular}

Os fluidos newtonianos são caracterizados pela proporcionalidade entre tensão cisalhante e taxa de cisalhamento, de modo que a sua viscosidade seja única e absoluta para dada temperatura, não dependendo do gradiente de velocidade. Além disso, foi possível observar que a viscosidade é fortemente influenciada pela temperatura, pois com o aumento da temperatura, ocorreu a diminuição da viscosidade, visto que a agitação das moléculas de maneira desordenada aumenta a distância entre as micelas adjacentes; a variação na salinidade dos sistemas microemulsionados, porém, não provocou mudanças consideráveis no comportamento reológico. 


\section{CONCLUSÃO}

Da análise reológica dos sistemas estudados, foi possível concluir que as microemulsões, classificadas como fluidos newtonianos, apresentaram valores médios de viscosidade semelhantes aos dos fluidos utilizados para recuperação avançada de petróleo, principalmente nas temperaturas médias dos poços de petróleo, entre 40 a $60{ }^{\circ} \mathrm{C}$.

\section{REFERÊNCIAS}

BUDHATHOKI, M., BARNEE, S., SHIAU, B., HARWELL, J. Improved oil recovery by reducing surfactant adsorption with polyelectrolyte in high saline brine Colloids and Surfaces A: Physicochemical and Engineering Aspects, 498: 66-73, 2016.

CURBELO, F. D. S., SANTANNA, V. C., BARROS NETO, E. L., DUTRA JUNIOR, T. V., CASTRO DANTAS, T. N., DANTAS NETO, A. A., GARNICA, A. I. C. Adsorption of nonionic surfactants in sandstone. Colloids and Surfaces A: Physicochemical and Engineering Aspects, 293:1-4, 2007.

CURBELO, F. D. S., BARROS NETO, E. L., DUTRA JUNIOR, T. V., CASTRO DANTAS, T. N., GARNICA, A. I. C. Oil recovery performance of surfactant solutions and adsorption in sandstone. Petrol. Sci. Technol. 26:77-90, 2008.

KUMAR, S., MANDAL, A. Studies on interfacial behavior and wettability change phenomena by ionic and nonionic surfactants in presence of alkalis and salt for enhanced oil recovery, Applied Surface Science, vol. 372, pag. 42-51, 2007.

LIMA, N.M. Métodos de Recuperação em Reservatórios Carbonáticos. Monografia de graduação em Engenharia de Petróleo. Universidade Federal Fluminense, 2013.

SANTOS, F. K. G.; ALVES, J. V. A.; CASTRO DANTAS, T. N.; DANTAS NETO, A. A.; DUTRA JUNIOR, T. V.; Neto, E. L. B. Seleção de tensoativos não iônicos para uso na recuperação avançada de petróleo. $4^{\circ}$ PDPETRO, Campinas, SP v.2, p.21-24, 2007.

\section{AGRADECIMENTOS}

À UFPB e ao CNPq pelo apoio financeiro. 